\title{
Enema ion compositions for enhancing colorectal drug delivery
}

Katharina Maisel ${ }^{1,2}$, Sumon Chattopadhyay ${ }^{1,3}$, Thomas Moench ${ }^{4,5}$, Craig Hendrix ${ }^{6}$, Richard Cone $^{1,4}$, Laura M. Ensign ${ }^{1,3,7,8^{*}}$, and Justin Hanes ${ }^{1-3,7-9^{*}}$

${ }^{1}$ Center for Nanomedicine, Johns Hopkins University School of Medicine, 400 N. Broadway, Baltimore, MD 21231 (USA)

${ }^{2}$ Department of Biomedical Engineering, Johns Hopkins University School of Medicine, 720 Rutland Avenue, Baltimore, MD 21205 (USA)

${ }^{3}$ Department of Chemical and Biomolecular Engineering, Johns Hopkins University, $3400 \mathrm{~N}$. Charles Street, Baltimore, MD 21218 (USA)

${ }^{4}$ Department of Biophysics, Johns Hopkins University, 3400 N. Charles Street, Baltimore, MD 21218 (USA)

${ }^{5}$ ReProtect, Inc, 703 Stags Head Road, Baltimore, MD 21286 (USA)

${ }^{6}$ Department of Clinical Pharmacology, Johns Hopkins University School of Medicine, $600 \mathrm{~N}$. Wolfe Street, Baltimore, MD 21287 (USA)

${ }^{7}$ Department of Ophthalmology, The Wilmer Eye Institute, Johns Hopkins University School of Medicine, 400 N. Broadway, Baltimore, MD 21231 (USA)

${ }^{8}$ Center for Cancer Nanotechnology Excellence, and the Institute for NanoBioTechnology, Johns Hopkins University, 3400 N Charles Street, Baltimore, MD 21218 (USA)

${ }^{9}$ Departments of Neurosurgery, Oncology, and Pharmacology \& Molecular Sciences, Johns Hopkins University School of Medicine, 600 N. Wolfe Street, Baltimore, MD 21287 (USA)

"co-corresponding authors

\section{Correspondence:}

Laura Ensign, PhD

lensign@jhmi.edu

or

Justin Hanes, PhD

hanes@jhmi.edu

443-287-7921

400 N. Broadway St., 6th Floor

Baltimore, MD 21231

\section{Keywords:}

microbicide, colon, pre-exposure prophylaxis, gastrointestinal tract 


\section{Abstract}

Delivering drugs to the colorectum by enema has advantages for treating or preventing both local and systemic diseases. However, the properties of the enema itself are not typically exploited for improving drug delivery. Sodium ions are actively pumped out of the lumen of the colon, which is followed by osmotically-driven water absorption, so we hypothesized that this natural mechanism could be exploited to drive nanoparticles and drugs to the colorectal tissue surface. Here, we report that sodium-based, absorption-inducing (hypotonic) enemas rapidly transport hydrophilic drugs and non-mucoadhesive, mucus penetrating nanoparticles (MPP), deep into the colorectal folds to reach virtually the entire colorectal epithelial surface. In contrast, isotonic and secretion-inducing (hypertonic) vehicles led to non-uniform, poor surface coverage. Sodium-based enemas induced rapid fluid absorption even when moderately hyperosmolal ( $350 \mathrm{mOsm}$ ) compared to blood ( $300 \mathrm{mOsm}$ ), which suggests that active sodium absorption plays a key role in osmosis-driven fluid uptake. We then used tenofovir, an antiretroviral drug in clinical trials for preventing HIV, to test the effects of enema composition on local and systemic drug delivery. We found that strongly hypotonic and hypertonic enemas caused rapid systemic drug uptake, whereas moderately hypotonic enemas with ion compositions similar to feces resulted in high local tissue levels with minimal systemic drug exposure. Similarly, moderately hypotonic enemas provided improved local drug retention in colorectal tissue, whereas hypertonic and isotonic enemas provided markedly reduced drug retention in colorectal tissue. Lastly, we found that moderately hypotonic enema formulations caused little to no detectable epithelial damage, while hypertonic solutions caused significant damage, including epithelial sloughing; the epithelial damage caused increased systemic drug absorption and penetration of MPP into colorectal tissue, a potential advantage in certain drug delivery applications. In summary, we illustrate that enema composition can be adjusted to maximize local versus systemic drug delivery, and that mildly hypotonic, sodium-based vehicles can provide uniform drug and MPP delivery in the colon that maximizes local drug concentrations.

317 words 


\section{Introduction}

Drug delivery by enema is the method of choice for treating and preventing local diseases, such as inflammatory bowel disease (IBD) and preventing sexually transmitted infections (STI) [1-4]. In addition, colorectal drug delivery can provide increased local tissue drug concentrations without systemic exposure to large drug doses taken orally [3,5]. Systemic drug delivery via the colorectum is commonly used in children and adults when drugs are either poorly bioavailable or oral ingestion is not possible [3-6]. Colorectal delivery allows drugs to reach systemic circulation without degradation due to stomach acid, digestive enzymes, and hepatic first-pass metabolism. Stable, water-soluble drugs can be administered directly to the colorectum in aqueous enemas. Poorly water soluble and/or easily degraded drugs can be formulated into nanocrystals or nanoparticles, and then suspended into aqueous enemas. In either case, the enema vehicle itself, the importance of which is often neglected, can have a major impact on colorectal drug delivery. More effective drug delivery locally to the colorectum has the potential to improve therapy for diseases with local manifestations, such as inflammatory bowel disease (IBD) and colorectal cancer, and for preventing colorectally transmitted infections such as HIV. In addition, the rational design of enema formulations can improve systemic bioavailability of drugs poorly absorbed after oral administration.

Many functions of the gastrointestinal (GI) tract utilize bulk fluid flow: in the small intestine, many liters of fluid are absorbed daily to rapidly transport nutrients through the mucus barrier covering the villi, and in the colon, additional water is withdrawn to dry the feces [7, 8]. Thus, we hypothesized that fluid absorption by the colorectum could be harnessed for more effective delivery of small molecules and nanoparticles. However, drugs or drug-loaded nanoparticles must pass through the "filter" formed by highly viscoelastic and adhesive layers of mucus coating the GI tract epithelium, or be trapped and rapidly cleared along with the 'sloppy' layers of mucus farthest from the epithelium [9, 10]. We previously found that nanoparticles sufficiently small and well-coated to slip through the mucus barrier, mucus-penetrating particles (MPP), can be efficiently delivered to the entire colorectal epithelial surface [11-14]. Rapid and widespread distribution of MPP was driven by the rapid absorption of water from hypotonic solution "advectively" transporting MPP to the epithelium much faster than by diffusion alone [11]. Similarly, we found that hypotonic vehicles induced fluid absorption by the vaginal epithelium that rapidly delivered water-soluble drugs and MPP to the epithelial surface by advection $[15,16]$. In contrast, MPP administered in isotonic vehicles (no fluid absorption) remained in the vaginal lumen and only slowly diffused through the mucosal barrier [15]. Thus, MPP provide a simple and sensitive method for observing the direction of fluid movement induced by the tonicity of the vehicle, as well as serving as carriers for mucosal drug delivery.

Here, we investigated the impact of enema composition on fluid absorption/secretion and drug delivery in the colorectum. We explored a range of solutions, hypotonic (absorptioninducing vehicles such as pure water, $20 \mathrm{mOsm}$ ) to hypertonic (secretion-inducing vehicles such as Fleet ${ }^{\circledR},>2200 \mathrm{mOsm}$ ), as well as solutions that contained sodium or potassium ions. We found that the tonicity of a solution depends not only on the osmolality, but also on the ion composition owing to specific ion transport processes of the colonic epithelium. Further, the properties of the enema and their effect on the mucosal environment can be harnessed to selectively enhance local tissue absorption versus systemic absorption. We demonstrate that a 
moderately hypotonic, sodium-based enema formulated with ion concentrations similar to those in feces (simulated colon solution, or SCS), provided enhanced colorectal distribution of both MPP and water soluble drug, improved tissue uptake of water-soluble drug, and caused no detected toxicity in the colorectum. In addition, we show that hypertonic enemas caused tissue damage that facilitated systemic drug absorption, and if used as a pretreatment, nanoparticle penetration into colorectal tissue. Our investigations illustrate the ability to tune enema composition to improve local and/or systemic drug delivery via the colorectum.

\section{Materials and Methods}

\section{Animal Model}

Rodents are commonly used as animal models for delivery to the Gl tract. However, rodents defecate much more frequently (every 5-10 min for mice) than is typical for humans, and the hard, desiccated pellets do not resemble human feces. To simulate the softer stool consistency and less frequent defecation rate of humans, female 6-8 week old CF-1 mice (Harlan) were starved for $24 \mathrm{~h}$. Mice were housed in cages with wire bottoms to prevent coprophagia. Mice were anesthetized with the drop-method via isoflurane for shorter procedures, or injected with $300 \mathrm{mg} / \mathrm{kg}$ Avertin (2, 2, 2-Tribromoethanol), using a $20 \mathrm{mg} / \mathrm{mL}$ working solution in phosphate buffered saline (PBS), when more extended anesthesia times were required. The various test solutions were administered intrarectally to mice with a Wiretrol (Drummond, Inc.) at volumes of $20 \mu \mathrm{L}$. All experimental procedures were approved by the Johns Hopkins Animal Care and Use Committee.

\section{Nanoparticle formulation and characterization}

MPP were synthesized as previously described [17]. Briefly, fluorescently labeled carboxylatemodified polystyrene (PS-COOH) nanoparticles, with manufacturer-listed sizes of 40 or $200 \mathrm{~nm}$ in diameter, were purchased from Molecular Probes (Eugene, OR). To obtain a dense polyethylene glycol (PEG) coating, nanoparticles were suspended in $200 \mathrm{mM}$ borate buffer, and $5 \mathrm{kDa}$ methoxy-PEG-amine was added in excess. N-Hydroxysulfosuccinimide (NHS, Sigma) and 1-Ethyl-3-(3-dimethylaminopropyl) carbodiimide (EDC, Invitrogen) were subsequently added to couple the carboxylic acid on the nanoparticles with the amine group on the PEG. Nanoparticles were suspended in $10 \mathrm{mM} \mathrm{NaCl}$ solution to measure size and $\zeta$-potential via dynamic light scattering $\left(90^{\circ}\right.$ scattering angle) and laser Doppler anemometry, respectively, using a Zetasizer Nano ZS90 (Malvern Instruments). Measurements were taken at $25^{\circ} \mathrm{C}$ according to instrument settings. A near-neutral $\zeta$-potential indicated sufficient PEG surface coverage to make nanoparticles that penetrate mouse colorectal mucus (MPP), as previously described [11]. MPP were either $60 \pm 1 \mathrm{~nm}$ or $230 \pm 4 \mathrm{~nm}$ in (hydrodynamic) diameter with $\zeta$ potentials of $-2.2 \pm 0.2 \mathrm{mV}$ and $-1.9 \pm 0.2 \mathrm{mV}$, respectively (before PEGylation the sizes were 56 $\pm 2 \mathrm{~nm}$ or $190 \pm 3 \mathrm{~nm}$, and $\zeta$-potentials were $-33 \pm 1 \mathrm{mV}$ or $-53 \pm 2 \mathrm{mV}$, respectively). Nanoparticles were confirmed to diffuse in human cervicovaginal mucus via multiple particle tracking (data not shown), which is also indicative of their ability to diffuse in mouse colorectal mucus [18].

\section{Enema formulations}


To obtain sodium-based solutions of various osmolalities, concentrated (10x) tris-buffered saline (Mediatech, Holly Hill, FL) was diluted with DI water. Similarly, to obtain potassium-based solutions $\left(\mathrm{KPO}_{4}\right)$ of various osmolalities, potassium phosphate buffer $\left(1 \mathrm{M} \mathrm{K}_{2} \mathrm{HPO}_{4} \mathrm{pH} 5.5\right.$, Sigma, St. Louis, MO) was diluted with DI water (e.g., 150 mOsm buffer was made by diluting $1 \mathrm{M} \mathrm{K}_{2} \mathrm{HPO}_{4} \sim 12$ fold). The simulated colon solution (SCS) was made by dissolving sodium bicarbonate, potassium chloride, dibasic potassium phosphate and monobasic potassium phosphate (Sigma, St. Louis, MO) in DI water at the following ion concentrations: $75 \mathrm{mM} \mathrm{K}^{+}, 25$ $\mathrm{mM} \mathrm{Na}^{+}, 35 \mathrm{mM} \mathrm{Cl}^{-}, 30 \mathrm{mM} \mathrm{PO}_{4}{ }^{3-}$, and $25 \mathrm{mM} \mathrm{CO}_{3}{ }^{2-}$ [19]. Fleet ${ }^{\circledR}$ enema solution and Fleet $^{\circledR}$ Naturals were purchased over the counter. All enema solutions were sterile-filtered through a $0.2 \mu \mathrm{m}$ filter prior to use in vivo. A $5 \% \mathrm{w} / \mathrm{v}$ glycerol gel was obtained by mixing the universal placebo hydroxyethylcellulose (HEC) gel (ReProtect, Inc., Baltimore, MD) with glycerol. Solution osmolality was measured with a vapor pressure osmometer (Wescor Vapro, Logan, UT) and measurements were reported as mean \pm SEM for $n=3$ measurements. The glycerol gel and Fleet ${ }^{\circledR}$ were serially diluted in DI water prior to measurement, and the osmolality was calculated by extrapolation of the obtained data points. Ion concentrations and osmolalities of the various formulations are listed in Table 1.

Table 1: Ion concentrations and osmolalities of various formulations. Osmolalities were measured using a vapor pressure osmometer and are reported as mean \pm SEM of $n=3$ measurements.

\begin{tabular}{|c|c|c|c|c|c|c|c|c|}
\hline \multirow[b]{2}{*}{ Formulation } & \multicolumn{8}{|c|}{ Composition } \\
\hline & $\begin{array}{l}\mathrm{Na}^{+} \\
(\mathrm{mM})\end{array}$ & $\begin{array}{c}\mathrm{K}^{+} \\
(\mathrm{mM})\end{array}$ & $\begin{array}{l}\mathrm{PO}_{4}^{3-} \\
(\mathrm{mM})\end{array}$ & $\begin{array}{c}\mathrm{Cl}^{-} \\
(\mathrm{mM})\end{array}$ & $\begin{array}{l}\mathrm{CO}_{3}{ }^{2-} \\
(\mathrm{mM})\end{array}$ & $\begin{array}{c}\text { Glycerol } \\
(w / v)\end{array}$ & $\begin{array}{l}\text { HEC } \\
(\mathrm{Y} / \mathrm{N})\end{array}$ & $\begin{array}{l}\text { Osmolality } \\
\text { (mOsm) }\end{array}$ \\
\hline $1 \times$ TBS & 138 & 0 & 0 & 138 & 0 & 0 & $\mathrm{~N}$ & $260 \pm 20$ \\
\hline scs & 25 & 75 & 30 & 35 & 25 & 0 & $\mathrm{~N}$ & $150 \pm 1$ \\
\hline SCS $\mathrm{K}^{+}$only & 0 & 75 & 30 & 35 & 0 & 0 & $\mathrm{~N}$ & $131 \pm 1$ \\
\hline SCS, $\mathrm{Na}^{+}$only & 25 & 0 & 0 & 0 & 25 & 0 & $\mathrm{~N}$ & $55 \pm 1$ \\
\hline 1.5x TBS & 205 & 0 & 0 & 205 & 0 & 0 & $\mathrm{~N}$ & $450 \pm 2$ \\
\hline $5 \%$ glycerol & 0 & 0 & 0 & 0 & 0 & 5 & $\mathrm{~N}$ & $600 \pm 2$ \\
\hline $5 \%$ glycerol gel & 150 & 0 & 0 & 150 & 0 & 5 & Y & $760 \pm 3$ \\
\hline Fleet $^{\circledR}$ enema & $\sim 1600$ & 0 & $\sim 1400$ & 0 & 0 & 0 & $\mathrm{~N}$ & $\geq 2200 \pm 1$ \\
\hline Fleet naturals ${ }^{\circledR}$ & NA & 0 & 0 & NA & 0 & 0 & $\mathrm{~N}$ & $260 \pm 3$ \\
\hline
\end{tabular}


Nanoparticle and free drug distribution on tissues

The $60 \mathrm{~nm}$ MPP were diluted 1:10 in TBS and $\mathrm{KPO}_{4}$ buffers of various osmolalities to make a range of sodium-based and potassium-based buffered solutions. To observe the colorectal distribution of the anti-retroviral drug, tenofovir (TFV), fluorescein isothiocyanate (FITC) was covalently reacted to the amino group on its purine ring. FITC-labeled TFV (FITC-TFV) was then dissolved 1:10 with unmodified TFV in the various solutions at an overall concentration of $1 \%$ $(\mathrm{w} / \mathrm{v})$, which is the concentration of TFV tested in the CAPRISA 004 clinical trial [20]. Mice were anesthetized using isoflurane during administration to ensure that the enema fluids would not be immediately expelled. To obtain cross-sectional images for assessing qualitative colorectal distribution, $20 \mu \mathrm{L}$ of solution was administered intrarectally to mice. The mice were sacrificed after 5-10 min. For distribution post Fleet ${ }^{\circledR}$ enema, mice were anesthetized with isoflurane and $200 \mu \mathrm{L}$ of Fleet ${ }^{\circledR}$ was administered intrarectally. After 10-15 min to allow for fluid expulsion (confirmed visually), $20 \mu \mathrm{L}$ of 60 or $230 \mathrm{~nm}$ MPP diluted 1:10 in DI water was administered. Mice were sacrificed after 5-10 min. Segments of tissue 1-2 cm in length were flash-frozen in Optimal Cutting Temperature (OCT) compound. Transverse sections of tissue $6 \mu \mathrm{m}$ thick were cut at various points along the length of the colorectum using a Leica CM-3050-S cryostat. Sections containing nanoparticles were fixed with formalin and stained for cell nuclei using ProLong Gold antifade reagent with DAPI (Invitrogen). Sections containing free drug were not fixed to reduce quenching of the fluorophore. All sections were stained with ProLong Gold antifade reagent with DAPI. Fluorescent images were obtained using an inverted epifluorescence microscope (Zeiss Axio Observer). To quantify nanoparticle distribution, one representative section was chosen per animal, and an outline of the epithelial perimeter was drawn. The percent overlap between the perimeter and fluorescence (\% colocalization of nanoparticles with the epithelial perimeter) due to the presence of nanoparticles was then calculated for each image using the JACoP plugin for ImageJ, and the average and standard error of the mean (SEM) was reported for $\mathrm{n} \geq 3$ mice.

Free drug uptake into plasma after administration to the mouse colorectum Mice were anesthetized with Avertin, and $20 \mu \mathrm{L}$ of the various solutions containing $1 \% \mathrm{w} / \mathrm{v}$ unlabeled TFV and $1 \mu \mathrm{L}$ of $\mathrm{H}^{3}$-TFV $(1 \mathrm{mCi} / \mathrm{mL}$, suspended in ethanol, $>97 \%$ pure, Moravek Biochemicals) was administered intrarectally. For the $t=0$ time point, retro-orbital blood collection was used to collect blood immediately after administration of each solution; for the 30 min time point, blood was collected 30 min post administration. Whole blood was centrifuged in heparinized tubes to obtain plasma, and $200 \mu \mathrm{L}$ of plasma was dissolved in $5 \mathrm{~mL}$ of Solvable ${ }^{\mathrm{TM}}$ and bleached using hydrogen peroxide. $500 \mu \mathrm{L}$ of plasma/Solvable ${ }^{\mathrm{TM}}$ solution was added to 10 $\mathrm{mL}$ of Ultima Gold, and $\mathrm{H}^{3}$ content was analyzed using a scintillation counter. Concentrations were calculated using a calibration curve of free $\mathrm{H}^{3}$-TFV. Separate standard curves for high and low count samples were made by serial dilution of $\mathrm{H}^{3}$-TFV. The concentrations of $\mathrm{H}^{3}-\mathrm{TFV}$ in the plasma samples were estimated using the standard curves, and the total drug concentration was calculated assuming that the $\mathrm{H}^{3}$-TFV and TFV were absorbed similarly in the colorectum.

Pharmacokinetic studies of free drug in the mouse colorectal tissue Mice were anesthetized with Avertin, and $20 \mu \mathrm{L}$ of the various solutions containing $1 \%(\mathrm{w} / \mathrm{v})$ unlabeled TFV and $1 \mu \mathrm{L} \mathrm{H} \mathrm{H}^{3}$-TFV (1 $\mu \mathrm{Ci} /$ mouse) was administered intrarectally to mice. 
Colorectal tissue was harvested at $5 \mathrm{~min}, 30 \mathrm{~min}, 1 \mathrm{~h}, 2 \mathrm{~h}$, and $4 \mathrm{~h}$ and processed as described above. $\mathrm{H}^{3}$ tissue content was measured using a scintillation counter and normalized based on individual tissue weights. Values were obtained from $n=5$ mice, and the highest and lowest values were removed from each group to obtain $n=3$ per time point for each solution used. The total drug concentration was calculated assuming that the $\mathrm{H}^{3}$-TFV and TFV were absorbed similarly by the colorectum.

\section{Toxicity of enema formulations in the mouse colorectum}

Mice were anesthetized with Avertin, and $50 \mu \mathrm{L}$ of sterile-filtered tap water (similar osmolality to DI water, 20 mOsm DI water v. 40 mOsm tap water), SCS, or Fleet ${ }^{\circledR}$ was administered as an enema. Mice were kept in supine position for 15-30 min prior to sacrifice and excising of tissues. For histological analysis, tissues were fixed in formalin and taken to the Johns Hopkins Medical Institutions Reference Histology Laboratory for standard paraffin embedding, sectioning, and hematoxylin and eosin (H\&E) staining. Images were obtained using a light microscope with a $10 \times / 0.25$ NA objective (Nikon E600).

\section{Results}

\section{Effect of enema osmolality and ion composition on colorectal MPP distribution}

First, we used MPP to probe the effects of enema osmolality on colorectal distribution. It was evident that sodium-based enemas below a certain osmolality (between 350-450 mOsm; 210 $\mathrm{mM} \mathrm{NaCl}$ ) induced fluid absorption that resulted in uniform MPP distribution over nearly the entire epithelial surface (Fig. 1A). In contrast, sodium-based enemas with osmolality $\geq 450$ mOsm caused fluid secretion and bowel distension, resulting in MPP remaining more centrally located in the lumen, rather than coming in close proximity to the epithelial surface (Fig. 1A; $450,600,860$, and 2200 mOsm). Next we quantified MPP surface coverage as a function of absorption- or secretion-inducing formulations. We found that MPP administered in sodiumbased enemas with osmolalities below 350-450 mOsm provided $>70 \%$ coverage of the colorectal epithelium, whereas MPP administered in solutions with an osmolality $\geq 450 \mathrm{mOsm}$ did not enter the epithelial folds, reducing their surface coverage to $<45 \%$, and as low as $0 \%$ when administered in a Fleet ${ }^{\circledR}$ enema (Fig. 1B). The coverage provided by all formulations below 350-450 mOsm was statistically significant compared to the coverage provided by all formulations $\geq 450 \mathrm{mOsm}$. Thus, we estimate that the effective isotonic point, which is the solution osmolality that does not induce fluid absorption nor secretion, for sodium-based enemas in the mouse colorectum lies between 350 and $450 \mathrm{mOsm}$.

Potassium, in contrast to sodium, is pumped from the tissues and into the lumen of the colorectum, creating a potassium gradient opposite to sodium and a high potassium/sodium ratio in feces [19]. This led us to hypothesize that increasing the potassium concentration in an enema would slow fluid absorption by the epithelium. MPP administered in a $150 \mathrm{mOsm}$ potassium buffer, markedly hypo-osmolal with respect to blood, would be expected on the basis of osmolality to induce fluid absorption, but instead this enema did not induce absorption (Fig. 2A). In contrast, a 150 mOsm sodium-based enema induced rapid absorption that advectively 

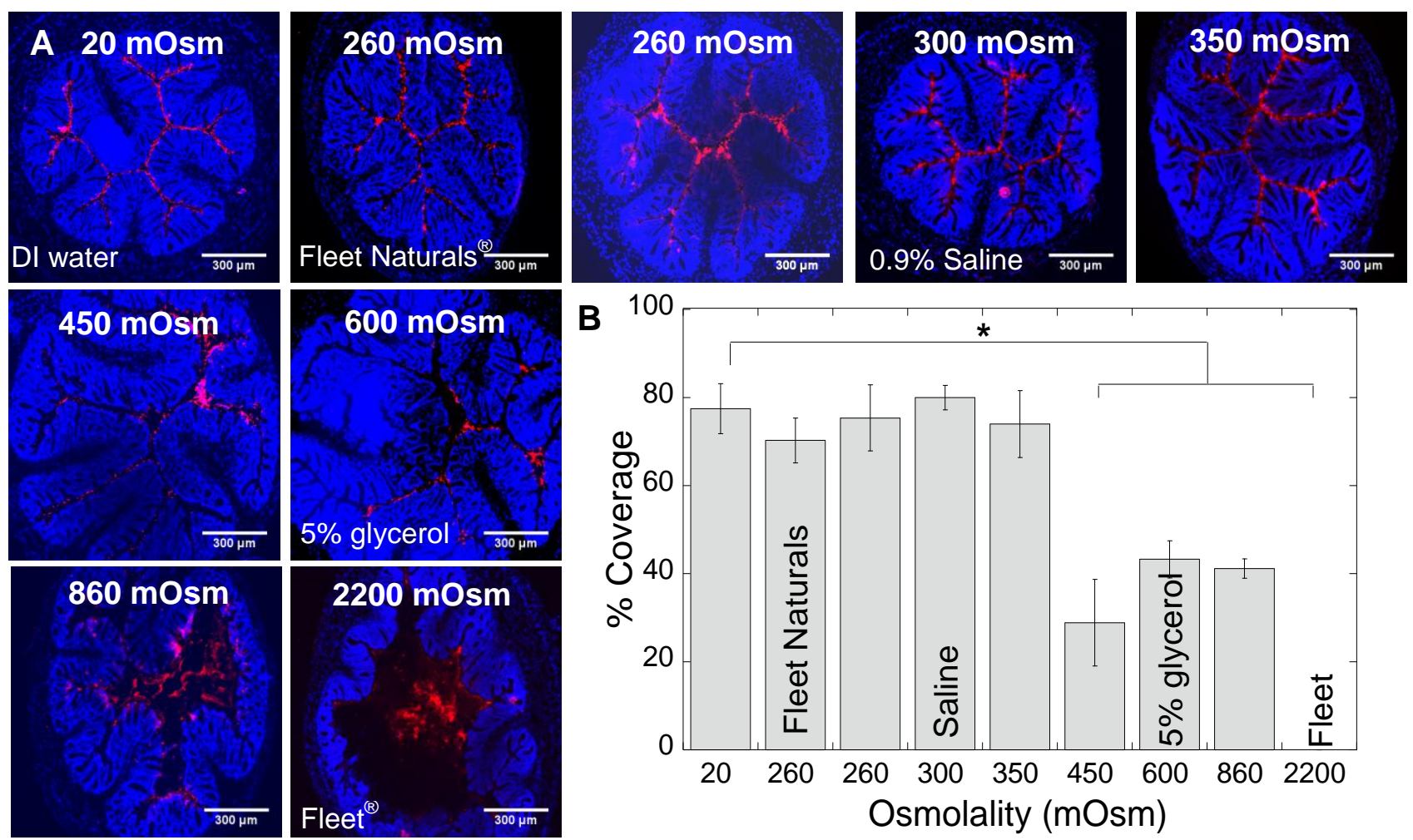

Figure 1. Distribution of MPP administered in sodium-based solutions with various osmolalities. (A) Distribution of $60 \mathrm{~nm}$ MPP in transverse colorectal cryosections 5-10 min after intrarectal administration in DI water (20 mOsm); sodium-based tris buffers (TBS) with osmolalities of $260,350,450$, and $860 \mathrm{mOsm} ; 5 \%$ glycerol in DI water (600 mOsm); $0.9 \%$ saline (300 mOsm, "normal saline"); Fleet Naturals ${ }^{\circledR}(260 \mathrm{mOsm})$; and regular Fleet ${ }^{\circledR}(2200 \mathrm{mOsm})$. Cell nuclei in transverse crysections were stained with DAPI. White scale bars $=$ $300 \mu \mathrm{m}$. Images are representative of $\mathrm{n} \geq 3$ mice. (B) Quantified surface coverage of MPP administered in various solutions on flattened mouse colorectal tissue. Data are calculated as means \pm SEM. ${ }^{*} P<0.05$ as compared to DI water (20 mOsm), Student's t-test.

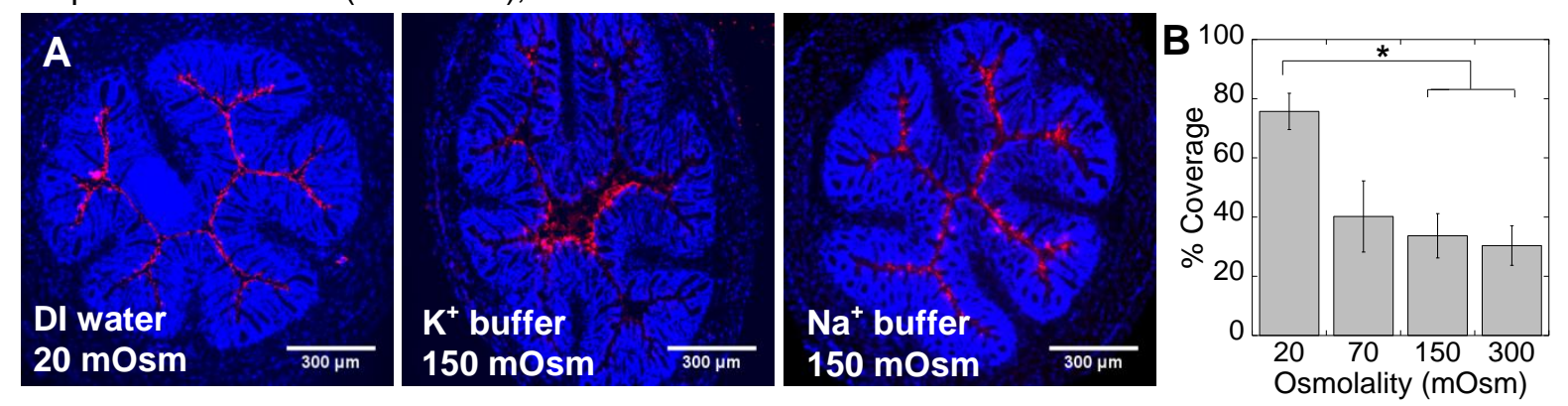

Figure 2. The impact of potassium on the colorectal distribution of MPP. (A) Distribution of $60 \mathrm{~nm}$ MPP in transverse colorectal cryosections 5-10 min after intrarectal administration in DI water (20 mOsm), potassium phosphate buffer (K+ buffer, $150 \mathrm{mOsm})$, and sodium-based tris buffer ( $\mathrm{Na}+$ buffer, 150 mOsm). Cell nuclei in transverse crysections are stained with DAPI. Images are representative of $n$ $\geq 3$ mice. White scale bars $=300 \mu \mathrm{m}$. (B) Quantified surface coverage of MPP administered in potassium phosphate buffers with various osmolalities on mouse colorectal tissue. The images in (A) are illustrative of the difference in distribution of MPP after administration in sodium and potassium based enemas, whereas the quantified surface coverage in $(B)$ is only for potassium-based solutions. Data are calculated as means \pm SEM. ${ }^{*} P<0.05$ as compared to DI water $(20 \mathrm{mOsm})$, Student's t-test.

transported MPP to uniformly coat the epithelial surface throughout the colorectum similarly to MPP administered in DI water (Fig. 2A). We estimate that the effective isotonic point (again, the 
point where there is no net flow of fluid) for potassium phosphate enemas is between 20 and 70 mOsm, since colorectal tissue coverage by MPP significantly decreased as the osmolality of the potassium-based enema solution increased above this range (Fig. 2B). As shown in Figures 1B and $2 \mathrm{~B}$, sodium-based enemas were significantly more effective for achieving maximal tissue coverage by MPP.

\section{Effect of osmolality of sodium-based solutions on colorectal drug distribution}

We demonstrated previously that hypotonic solutions may also be advantageous for improving the distribution of water soluble drugs in the mouse vagina, leading to uniform drug delivery to the entire epithelial surface [15]. Here, we explored colorectal delivery of TFV in a hypotonic enema as a method for protection against HIV by pre-exposure prophylaxis (PrEP). We first compared sodium-based enemas of varying osmolality: DI water as hypotonic control (20 mOsm), 0.9\% saline (300 mOsm; "normal saline"), TBS buffer at the osmolality in the isotonic range for the colorectum (350-450 mOsm), and slightly hypertonic TBS buffer (650 mOsm) that was expected to induce fluid secretion. As shown in Figure 3, fluorescentlylabeled TFV distribution was qualitatively consistent with MPP distribution in the various vehicles: colorectal tissue coverage (and therefore access to target cells and tissues) was
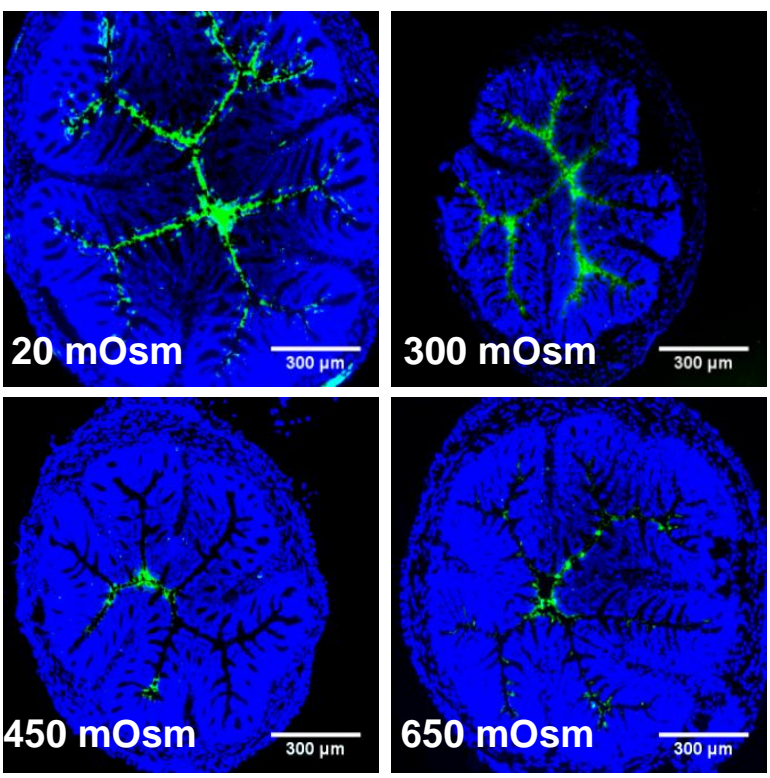

Figure 3. Distribution of TFV administered in sodium-based solutions with various osmolalities. Distribution in transverse colorectal cryosections 5-10 min after intrarectal administration of $1 \%$ TFV (1:10 TFV-FITC:TFV) in sodium-based tris buffers (450 and $650 \mathrm{mOsm})$, DI water (20 mOsm), and $0.9 \%$ saline (300 mOsm). Cell nuclei are stained with DAPI. Images are representative of $n \geq 3$ mice. White scale bars $=$ $300 \mu \mathrm{m}$. improved by using an absorption-inducing enema. Bulk fluid flow rapidly transported the watersoluble small molecule drug through the mucus barrier and to the epithelium, and also transported the drug deep into the collapsed in folds of the colorectum.

We then

formulated an enema that has sodium and potassium concentrations similar to those found in feces, which is likely to provide safe water absorption in the colorectum. The osmolality of this SCS
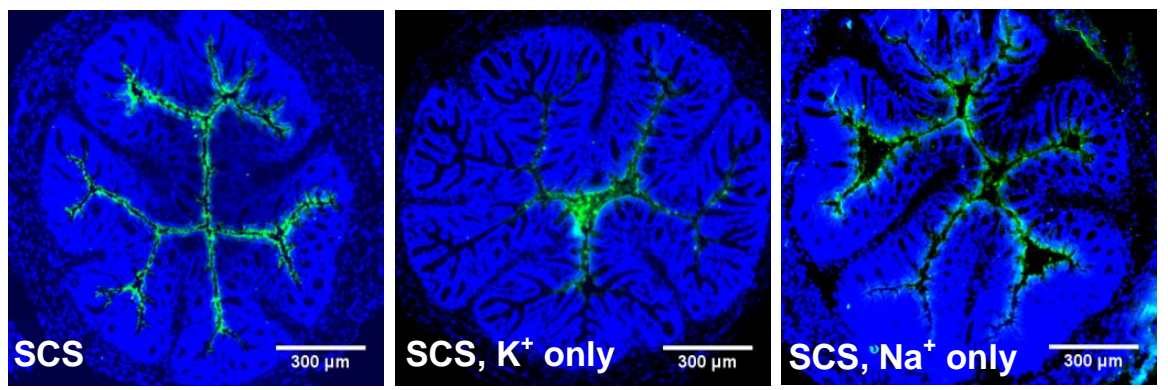

Figure 4. Distribution of TFV in SCS, SCS with only potassium concentration matched to that of feces, and SCS with only sodium concentration matched to that of feces. Distribution in transverse colorectal cryosections 5-10 min after intrarectal administration of 1\% TFV (1:10 TFV-FITC:TFV) in SCS, SCS without sodium (SCS, K+ only) and SCS without potassium (SCS, Na+ only). Cell nuclei in transverse cryosections are stained with DAPI. Images are representative of $n \geq 3$ mice. White scale bars $=300 \mu \mathrm{m}$. 
formulation was approximately 150 mOsm (Table 1). Since the solution contained both sodium $(25 \mathrm{mM})$ and potassium $(75 \mathrm{mM})$, we investigated the individual contributions of these ions to bulk fluid flow. We found that fluid absorption only occurred if sodium was present in the solution (Fig. 4). If sodium was removed, leaving potassium at $75 \mathrm{mM}$ (SCS, $\mathrm{K}^{+}$only), no fluid absorption occurred, and TFV-FITC remained in the lumen. In contrast, if potassium was removed, leaving sodium at $25 \mathrm{mM}$ (SCS, $\mathrm{Na}^{+}$only), fluid was rapidly absorbed by the colorectal epithelium, distributing TFV-FITC throughout all of the folds of the mouse colorectum (Fig. 4).

\section{Effect of tonicity on colorectal drug uptake into plasma and tissue}

We previously described that very hypotonic delivery of a water soluble drug reduced vaginal retention, likely due to the drug being driven advectively all the way through the epithelium to reach systemic circulation [15]. Here, we investigated the effect of enema tonicity on movement of drugs from the colorectal lumen into the plasma. We compared drug delivered in a variety of solutions, including: DI water (20 mOsm), SCS (150 mOsm), near-isotonic TBS (450 mOsm), and the strongly hypertonic Fleet ${ }^{\circledR}$ enema (>2200 mOsm). In addition, we tested a mild secretion-inducing (hypertonic) solution containing $5 \%$ glycerol in DI water $(600 \mathrm{mOsm})$, which is the concentration of glycerol currently being used in the reduced-glycerol TFV gel formulation in clinical trials for colorectal PrEP [2, 21]. The results showed that no TFV Table 2: Plasma levels of TFV after administration in various enema solutions. Amount of TFV present in the plasma 0 min and 30 min after enema administration to mice. $1 \%$ TFV $\left(1: 100 \mathrm{H}^{3}\right.$-TFV:TFV) was administered in DI water (20 mOsm), SCS (150 mOsm), TBS (450 mOsm), 5\% glycerol (600 mOsm) and Fleet ${ }^{\circledR}$ was detected in the plasma after $30 \mathrm{~min}$ if it was administered in near-isotonic or moderately hypotonic SCS solution; however, ${ }^{3} \mathrm{H}$-TFV was found in the plasma 30 min after enema administration in very hypotonic (DI water), as well as very hypertonic $\left(\right.$ Fleet ${ }^{\circledR}$ ), vehicles (2200 mOsm) enema. Studies were performed in $n \geq 5$ mice.

\begin{tabular}{|c|c|c|c|c|}
\hline \multirow[b]{3}{*}{$\begin{array}{l}\text { Formulation } \\
\text { (osmolality) }\end{array}$} & \multicolumn{4}{|c|}{ Time after dosing } \\
\hline & \multicolumn{2}{|c|}{0 min } & \multicolumn{2}{|c|}{$30 \mathrm{~min}$} \\
\hline & TFV $(\mu \mathrm{g})$ & \# signal/\# total & TFV $(\mu \mathrm{g})$ & \# signal/\# total \\
\hline $\begin{array}{l}\text { DI water } \\
20 \text { mOsm }\end{array}$ & $0 \pm 0$ & $0 / 5$ & $7.4 \pm 3.4$ & $6 / 8$ \\
\hline $\begin{array}{c}\text { SCS } \\
150 \text { mOsm }\end{array}$ & $0 \pm 0$ & $0 / 5$ & $0 \pm 0$ & $0 / 5$ \\
\hline $\begin{array}{c}1.5 x \text { TBS } \\
450 \text { mOsm }\end{array}$ & $0 \pm 0$ & $0 / 5$ & $0 \pm 0$ & $0 / 5$ \\
\hline $\begin{array}{c}5 \% \text { Glycerol in DI water } \\
600 \text { mOsm }\end{array}$ & $0 \pm 0$ & $0 / 5$ & $0.1 \pm 0.1$ & $1 / 5$ \\
\hline $\begin{array}{l}\text { Fleet }^{\Theta} \text { enema } \\
2200 \text { mOsm }\end{array}$ & $0 \pm 0$ & $0 / 5$ & $42 \pm 18$ & $4 / 5$ \\
\hline
\end{tabular}

(Table 2).

To investigate how enema tonicity impacts drug levels in the tissue, we compared the amount of ${ }^{3} \mathrm{H}$-TFV associated with colorectal epithelial tissue when administered in SCS (150 mOsm), near-isotonic TBS (450 mOsm), and the strongly hypertonic Fleet ${ }^{\circledR}$ enema (>2200 mOsm). In addition, we tested the amount of ${ }^{3} \mathrm{H}$-TFV associated with colorectal epithelial tissue when administered in HEC gel containing $5 \%$ glycerol $(760 \mathrm{mOsm})$. Based on published cell 
migration data and the number of cell layers these cells need to ascend through the crypts in the mouse colorectum, we calculated that the surface epithelial layer, which would absorb drug, renews approximately every $2 \mathrm{~h}[22,23]$. For this reason, we assessed tissue concentration up to 4 $h$, at which time TFV tissue concentrations became low and indistinguishable between the different solutions (Fig. 5). The initial tissue TFV concentrations were highest when administered in Fleet ${ }^{\circledR}$ and in SCS compared to administration in near-isotonic (450 mOsm) TBS. But at $2 \mathrm{~h}$, tissue concentrations of TFV administered in Fleet $^{\circledR}$ or near-isotonic TBS were significantly lower than when administered in SCS (Fig. 5). The area under the curve (AUC) for TFV administered in SCS, nearisotonic 450 mOsm TBS, Fleet ${ }^{\circledR}$ enema, and $5 \%$ glycerol gel were 330 , 120,72 , and $21 \mu \mathrm{g} \cdot \mathrm{h} / \mathrm{g}$, respectively.

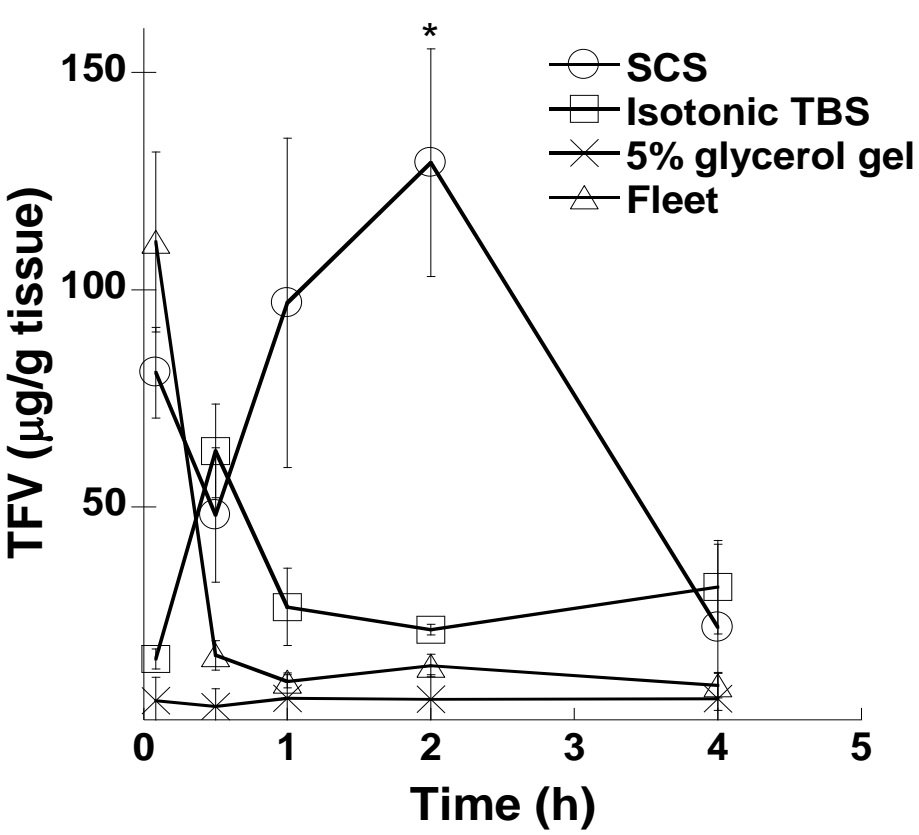

Figure 5: Pharmacokinetic analysis of TFV in mouse colorectal tissue. The calculated amount of TFV present in mouse colorectal tissues is shown for up to $4 \mathrm{~h}$ after administration. $1 \%$ TFV was administered in the simulated colon solution (SCS) (150 mOsm), isotonic TBS (450 mOsm), 5\% glycerol gel (760 mOsm), and Fleet ${ }^{\circledR}$ enema (2200 mOsm). Studies were performed in $n \geq 5$ mice with highest and lowest value excluded to yield a total of $n \geq 3$ sample points. Data is shown as mean \pm SEM. ${ }^{*} P<0.05$ using Student's t-test. Even though the $5 \%$ glycerol gel we used to mimic the gel in current PrEP clinical trials was only moderately hypertonic, the amount of TFV absorbed by the colorectal tissue was decreased by $>10$-fold compared to administration in the moderately hypotonic SCS.

\section{Toxicity of enema formulations}

Previous results have shown that hypertonic vehicles (gels, lubricants, enemas, etc.) are toxic to the human colorectal epithelium [24]. We investigated acute tissue effects of hypotonic and hypertonic enemas. We compared tap water (strongly hypotonic), strongly hypertonic Fleet ${ }^{\circledR}$, and our moderately hypotonic SCS enema in the mouse colorectum. As shown in Figure 6, Fleet ${ }^{\circledR}$ enema caused distension and epithelial disruption (red arrows) within 10 min of administration. In addition, the damage induced by the Fleet ${ }^{\circledR}$ enema allowed MPP, both 60 and $230 \mathrm{~nm}$ in size, to penetrate into the tissue regions where the epithelial integrity was compromised (Fig. 6B). Tap water and moderately hypotonic SCS did not induce any detected epithelial damage (Fig. 6A). 


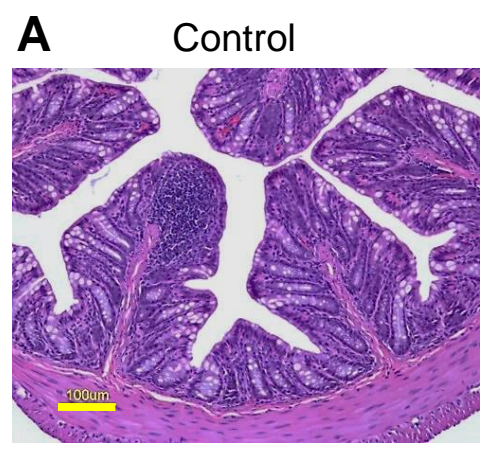

Simulated Colon Solution

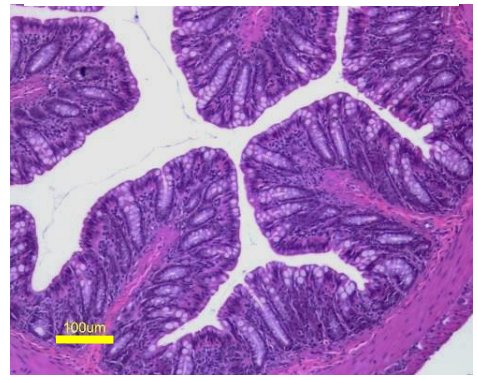

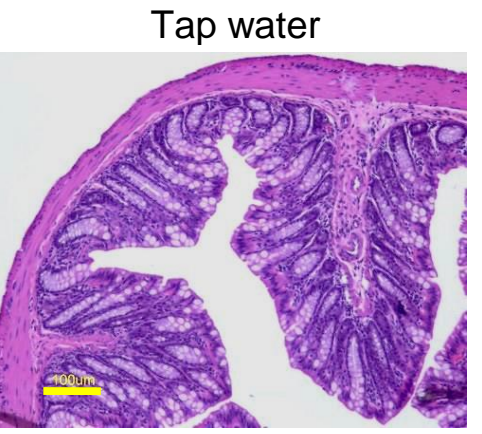

Fleet $^{\circledR}$
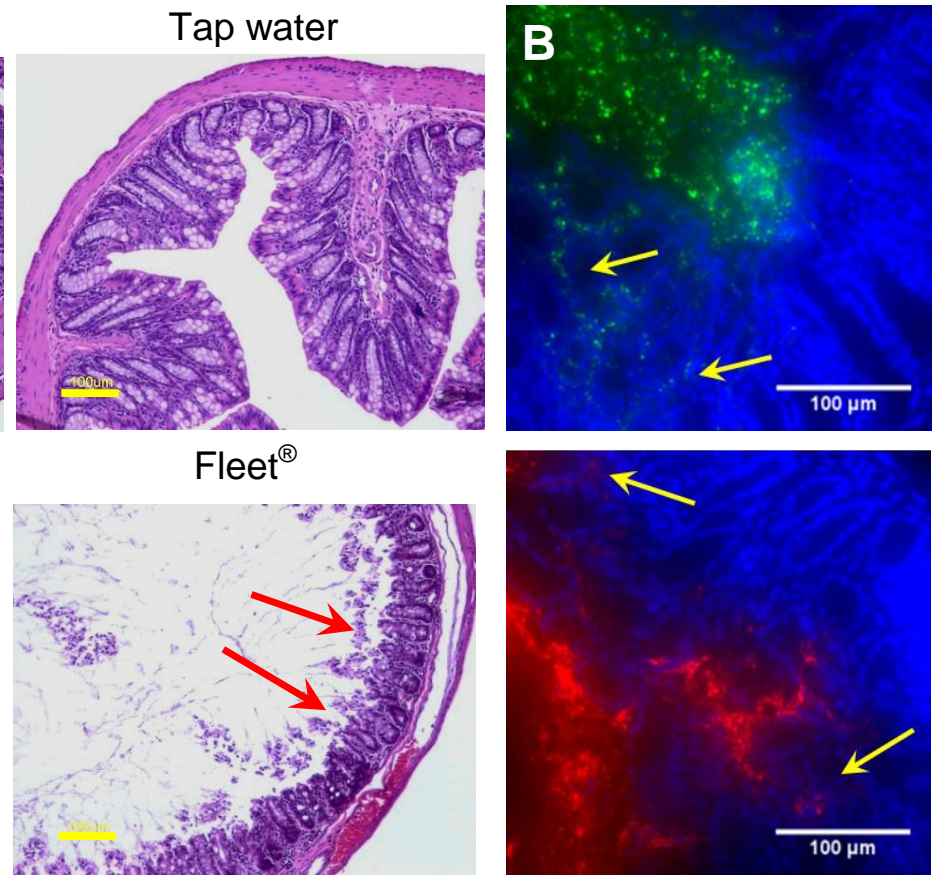

Figure 6. Acute toxicity of various enemas in the mouse colorectum. (A) Hemotoxylin and eosin (H\&E) stained mouse colorectal tissue excised 10-15 min after intrarectal administration of sterile-filtered tap water, simulated colon solution (SCS), or Fleet ${ }^{\circledR}$ enema. Red arrows indicate epithelial tissue that has separated from the tissue surface. Yellow scale bars $=100 \mu \mathrm{m}$. (B) Distribution of $60 \mathrm{~nm}$ (green) and $230 \mathrm{~nm}$ (red) MPP administered in strongly hypotonic DI water 10 min after pretreatment with Fleet $\AA^{\circledR}$ enema. Yellow arrows indicate areas where MPP have penetrated into the damaged tissue. White scale bars $=100 \mu \mathrm{m}$. All images are representative of $n \geq 3$ mice.

\section{Discussion}

The human colon absorbs 1.4-1.8 $\mathrm{L}$ of water every day, and this fluid uptake is driven by active ion transport [7]. Water absorption is accomplished by electrogenic $3 \mathrm{Na}^{+} / 2 \mathrm{~K}^{+}$ATPase pumps in the basolateral membranes, and high conductance sodium, potassium, and chloride channels in the apical membranes, of epithelial cells. This robust physiological mechanism provides an opportunity to use fluid absorption to achieve more effective colorectal drug delivery. We have previously demonstrated that MPP, sufficiently small and well-coated to rapidly diffuse through mouse colorectal mucus, provide improved colorectal distribution when administered hypotonically in the colorectum [11]. We have also previously found that small molecule drugs can be rapidly drawn into and through the epithelial layer in the vagina [15]. Thus, strongly hypotonic solutions, such as DI water, can cause drug to reach the systemic circulation (Table 2) $[15,25]$. Similarly, hypertonic solutions such as Fleet can also cause drug to reach systemic circulation (Table 2), likely as a result of extensive epithelial tissue damage [25]. Thus, to achieve systemic drug delivery, either strongly hypertonic or hypotonic solutions can be used for colorectal administration. However, fluid expulsion resulting from administration of a hypertonic solution may limit overall absorption in humans, and tissue damage by hypertonic vehicles may increase susceptibility to infections [26] . In contrast, a moderately hypotonic enema resulted in no detected systemic drug absorption (Table 2), but provided optimal colorectal tissue drug levels compared to isotonic and hypertonic formulations. In humans, Leyva and coworkers 
showed that systemic drug levels were highest when using water (strongly hypotonic) or $0.9 \%$ saline enema (300 mOsm, iso-osmolar to blood, but a formulation that likely causes moderate fluid absorption in the human colon due to active sodium ion uptake), while Fleet ${ }^{\circledR}$ (strongly hypertonic) resulted in significantly lower systemic drug absorption [25]. The difference in systemic absorption using Fleet ${ }^{\circledR}$ was likely due to the rapid fluid expulsion that occurs in humans post Fleet ${ }^{\circledR}$ enema administration, while rapid expulsion was prevented in mice since they were anesthetized during the experiments. Leyva and coworkers also reported that tissue concentrations were highest in the $0.9 \%$ saline (moderately hypotonic) enema, which correlates with our findings in mice [25].

For certain applications, systemic drug delivery via enema is desirable. Colorectal delivery has long been used to administer medications, such as anti-arthritic, anti-emetic and anti-angiogenic agents, to the systemic circulation [3-6]. Most of these medications have improved bioavailability when administered intrarectally, due to avoidance of first-pass metabolism in the liver and degradation by stomach acid and digestive enzymes. Our findings indicate that both strongly hypotonic (absorption-inducing) and hypertonic (secretion-inducing) enemas cause detectable systemic drug concentrations within $30 \mathrm{~min}$ of intrarectal administration in anesthetized mice. However, the toxicity induced by strongly hypertonic enemas in the colorectum may limit their utility in cases where epithelial toxicity would be detrimental (e.g. HIV PrEP). Leyva and coworkers also showed that hypertonic enemas caused epithelial injury in the human colorectum, while extremely hypo-osmolar (tap water) enema formulations did not [25]. Additionally, extremely hypotonic enemas may be able to transport drug through the epithelium by both paracellular and transcellular fluid absorption to the systemic circulation [27-29]. Leyva also suggested that strongly hypotonic enemas are favorable for systemic drug delivery, and intestinal epithelial cells show increased transport of hydrophilic molecules across the intestinal wall when the molecules are applied in a 50\% hypotonic solution (1/2 isotonic, or $75 \mathrm{mM} \mathrm{NaCl})[25,30]$. Therefore, a strongly hypotonic enema formulation may be optimal for systemic drug delivery via intrarectal administration.

When high tissue drug levels and low systemic drug exposure are desired, moderately hypotonic solutions may be optimal. These solutions can provide robust fluid flow through the protective mucus layers in the colon, resulting in increased local drug levels, and yet are mild enough to minimize systemic absorption. Sodium-based solutions drove fluid absorption in the colorectum of mice, as long as the concentration was below $205 \mathrm{mM} \mathrm{NaCl}$ (450 mOsm). This is similar to the results of Billich and coworkers, who infused different sodium-based solutions containing PEG as a non-absorbable marker into human colorectums using a peristaltic pump, and found that $220 \mathrm{mM} \mathrm{NaCl}$ solutions (theoretical osmolality: $440 \mathrm{mOsm}$ ) caused fluid secretion [31]. In addition, their experiments showed that sodium solutions iso-osmolar to blood $(150 \mathrm{mM} \mathrm{NaCl})$ resulted in fluid absorption in the human colon, which is consistent with our findings in the mouse colorectum [31]. We found that potassium, on the other hand, seemed to induce colorectal fluid secretion, even at very low concentrations/osmolalities. By varying the amounts of sodium and potassium, optimal concentrations may be found that lead to the highest local drug tissue levels. Here, we matched the sodium and potassium content typically found in feces, which we anticipated would have minimal toxic effects. However, excessive potassium absorption can also have systemic side effects, so careful study of the potential toxic effects of various enema ion compositions, particularly with repetitive use, is important for future 
development of enema products. Further, the interactions of specific drugs with the mucus lining and the epithelium can impact absorption [31-33]. For the water soluble drug tenofovir, we found that moderately advective absorption provided improved colorectal drug distribution and tissue uptake without excessive loss of drug to the systemic circulation (Table 2 and Figure 6). However, the duration of drug retention in the colorectal tissue was limited by the rapid turnover of the mouse colorectal epithelium, necessitating additional studies of the use of hypotonic enema vehicles in larger animals and humans.

In addition to diseases such as IBD, a particularly useful application for colorectal drug delivery is HIV PrEP. Risk of colorectal HIV transmission is $10-20$ fold higher than vaginal transmission, and enema use is widespread for cleansing the bowel prior to receptive anal intercourse (RAI) [1, 21, 34-39]. In addition to an increased risk of viral transmission with RAI, adherence to prescribed drug regimens has been a significant issue in most HIV PrEP clinical trials [40,41]. Using a microbicide-loaded enema would require minimal behavioral modification, leading to improved adherence, which may significantly improve PrEP outcomes. Currently available OTC enema formulations, like Fleet ${ }^{\circledR}$ and the TFV gels now in clinical trials [2, 24], are hypertonic in the colon, and thus induce secretion of fluid that likely opposes effective drug delivery, including significantly reducing epithelial distribution [25]. Moreover, Fleet ${ }^{\circledR}$ caused $^{-}$ epithelial damage that may significantly increase susceptibility to infection [24, 26, 42], as supported by the penetration of MPP even larger in size than HIV into the colorectal epithelium when administered after Fleet pretreatment (Fig. 6B). To provide maximal protection, microbicide drugs such as TFV should be effectively delivered to all of the susceptible cells and tissues, including deep into the folded surfaces of the colorectum, without inducing epithelial toxicity $[2,15,16]$. This is a task unlikely to be achieved with current microbicide formulations [2, $15,16]$. A moderately hypotonic enema may provide the desired uniform coverage of drug, or drug-loaded MPP, of the epithelial surface of the colorectum while minimizing epithelial damage. Although epithelial toxicity is highly undesirable in PrEP, it may be advantageous for other applications. A strongly hypertonic enema could be used as a pre-treatment that is followed later by hypotonically-delivered nanoparticles that could then penetrate deep into the damaged tissue (Fig. 6B), similar to the tissue penetration we have observed can occur with MPP (but not adhesive nanoparticles) in ulcerated gastrointestinal tissues [11]. Tissue penetration may, in turn, improve drug uptake into systemic circulation, as well as local tissue uptake and residence time. Enemas may thus be used as both pre-treatment and delivery vehicles to optimize local and/or systemic drug or nanoparticle delivery.

\section{Conclusion}

We report that hypotonic, sodium-based enemas improve the distribution of MPP and small water-soluble drugs on the colorectal epithelial surface compared with isotonic and hypertonic enemas and potassium-based enemas. We demonstrate that enema formulations can be tuned to deliver drugs into the local tissue or into systemic circulation by varying the ion content: hydrophilic free drug was rapidly taken up into systemic circulation when administered in strongly hypotonic or hypertonic solutions, while a moderately hypotonic enema (SCS) significantly improved local drug levels and reduced systemic exposure. We also illustrate that hypotonic enema formulations caused no detected epithelial damage, while hypertonic enemas 
significantly damaged the colorectal epithelium. When used as a pretreatment, damage caused by hypertonic enemas allowed penetration of non-adhesive MPP nanoparticles into the tissue.

\section{Acknowledgment}

We thank the animal husbandry staff at Johns Hopkins, the Wilmer Microscopy and Imaging Core Facility and the Nanotechnology Core (NIH grant \# P30EY001765). We also thank Dr. Brian Simons for expert consulting regarding assessment of colorectal epithelial toxicity. This work was supported by NIH grants R33AI094519, R33AI079740, U19AI133127, the Johns Hopkins University Center for AIDS Research (P30AI094189), the W.W. Smith Charitable Trust (grant A1302), and the NSF graduate research fellowship program. The mucus penetrating particle technology is being developed by Kala Pharmaceuticals. Dr. Hanes is a co-founder of Kala. Drs. Hanes and Cone own company stock, which is subject to certain restrictions under University policy. The terms of this arrangement are being managed by the Johns Hopkins University in accordance with its conflict of interest policies.

\section{References}

[1] A. Carballo-Dieguez, J. Bauermeister, A. Ventuneac, C. Dolezal, K. Mayer, Why rectal douches may be acceptable rectal-microbicide delivery vehicles for men who have sex with men, Sex Transm Dis, 37 (2010) 228-233.

[2] C.S. Dezzutti, L.C. Rohan, L. Wang, K. Uranker, C. Shetler, M. Cost, J.D. Lynam, D. Friend, Reformulated tenofovir gel for use as a dual compartment microbicide, J Antimicrob Chemother, 67 (2012) 2139-2142.

[3] K. Sugisaki, F. Honma, H. Iwadate, K. Shio, Y. Shioya, E. Fukaya, K. Sato, H. Saito, H. Sekine, H. Kobayashi, H. Orikasa, H. Watanabe, Y. Sato, Ulcerative colitis occurring in the course of rheumatoid arthritis: a case successfully treated with mesalamine enema, Intern Med, 43 (2004) 1046-1050.

[4] S. Szabo, X. Deng, G. Tolstanova, T. Khomenko, B. Paunovic, L. Chen, M. Jadus, Z. Sandor, Angiogenic and anti-angiogenic therapy for gastrointestinal ulcers: new challenges for rational therapeutic predictions and drug design, Curr Pharm Des, 17 (2011) 1633-1642.

[5] J. Herrstedt, M. Jorgensen, H.R. Angelo, M.R. Rassing, J. Moller-Sonnergaard, P.

Dombernowsky, Bioavailability of the antiemetic metopimazine given as a microenema, $\mathrm{Br} \mathrm{J}$ Clin Pharmacol, 41 (1996) 613-615.

[6] R. Baria, N. Joshi, D. Pandya, Clinical efficacy of Panchamuladi Kaala Basti (enema) in the management of Amavata (Rheumatoid Arthritis), Ayu, 32 (2011) 90-94.

[7] G.I. Sandle, Salt and water absorption in the human colon: a modern appraisal, Gut, 43 (1998) 294-299.

[8] E.W. Moore, Physiology of intestinal water and electrolyte absorption, in, American Gastroenterological Society, 1976.

[9] R.A. Cone, Barrier properties of mucus, Adv Drug Deliv Rev, 61 (2009) 75-85.

[10] L.M. Ensign, R. Cone, J. Hanes, Oral drug delivery with polymeric nanoparticles: the gastrointestinal mucus barriers, Adv Drug Del Rev, 64 (2012) 557-570.

[11] K. Maisel, L.M. Ensign, M. Reddy, R.A. Cone, J.S. Hanes, Effect of surface chemistry on nanoparticle interaction with gastrointestinal mucus and distribution in the gastrointestinal tract following oral and rectal administration in the mouse, $\mathrm{J}$ of Control Release, (2014). 
[12] S.K. Lai, D.E. O'Hanlon, S. Harrold, S.T. Man, Y.Y. Wang, R. Cone, J. Hanes, Rapid transport of large polymeric nanoparticles in fresh undiluted human mucus, Proc Natl Acad Sci, 104 (2007) 1482-1487.

[13] S.K. Lai, Y.Y. Wang, J. Hanes, Mucus-penetrating nanoparticles for drug and gene delivery to mucosal tissues, Adv Drug Deliv Rev, 61 (2009) 158-171.

[14] L.M. Ensign, C. Schneider, J.S. Suk, R. Cone, J. Hanes, Mucus penetrating nanoparticles: biophysical tool and method of drug and gene delivery, Adv Mater, 24 (2012) 3887-3894.

[15] L.M. Ensign, T.E. Hoen, K. Maisel, R.A. Cone, J.S. Hanes, Enhanced vaginal drug delivery through the use of hypotonic formulations that induce fluid uptake, Biomaterials, 34 (2013) 6922-6929.

[16] L.M. Ensign, B.C. Tang, Y.Y. Wang, T.A. Tse, T. Hoen, R. Cone, J. Hanes, Mucuspenetrating nanoparticles for vaginal drug delivery protect against herpes simplex virus, Sci Transl Med, 4 (2012) 138ra179.

[17] E.A. Nance, G.F. Woodworth, K.A. Sailor, T.Y. Shih, Q. Xu, G. Swaminathan, D. Xiang, C. Eberhart, J. Hanes, A dense poly(ethylene glycol) coating improves penetration of large polymeric nanoparticles within brain tissue, Sci Transl Med, 4 (2012) 149ra119.

[18] L.M. Ensign, A. Henning, C.S. Schneider, K. Maisel, Y.Y. Wang, M.D. Porosoff, R. Cone, J. Hanes, Ex vivo characterization of particle transport in mucus secretions coating freshly excised mucosal tissues, Mol Pharm, 10 (2013) 2176-2182.

[19] Units of Measurement, Body Fluid, Composition of Body, and Nutrition, 8 ed., Ciba-Geigy, 1981.

[20] Q. Abdool Karim, S.S. Abdool Karim, J.A. Frohlich, A.C. Grobler, C. Baxter, L.E. Mansoor, A.B. Kharsany, S. Sibeko, K.P. Mlisana, Z. Omar, T.N. Gengiah, S. Maarschalk, N. Arulappan, M. Mlotshwa, L. Morris, D. Taylor, Effectiveness and safety of tenofovir gel, an antiretroviral microbicide, for the prevention of HIV infection in women, Science, 329 (2010) 1168-1174. [21] I. McGowan, C. Dezzutti, Rectal Microbicide Development, Curr Top Microbiol Immunol, (2013).

[22] W.W. Chang, N.J. Nadler, Renewal of the epithelium in the descending colon of the mouse. IV. Cell population kinetics of vacuolated-columnar and mucous cells, Am J Anat, 144 (1975) 39-56.

[23] W.W. Chang, C.P. Leblond, Renewal of the epithelium in the descending colon of the mouse. I. Presence of three cell populations: vacuolated-columnar, mucous and argentaffin, Am J Anat, 131 (1971) 73-99.

[24] E.J. Fuchs, L.A. Lee, M.S. Torbenson, T.L. Parsons, R.P. Bakshi, A.M. Guidos, R.L. Wahl, C.W. Hendrix, Hyperosmolar sexual lubricant causes epithelial damage in the distal colon: potential implication for HIV transmission, J Infect Dis, 195 (2007) 703-710.

[25] F.J. Leyva, R.P. Bakshi, E.J. Fuchs, L. Li, B.S. Caffo, A.J. Goldsmith, A. Ventuneac, A. Carballo-Dieguez, Y. Du, J.P. Leal, L.A. Lee, M.S. Torbenson, C.W. Hendrix, Isoosmolar enemas demonstrate preferential gastrointestinal distribution, safety, and acceptability compared with hyperosmolar and hypoosmolar enemas as a potential delivery vehicle for rectal microbicides, AIDS Res Hum Retroviruses, 29 (2013) 1487-1495.

[26] T.R. Moench, R.J. Mumper, T.E. Hoen, M. Sun, R.A. Cone, Microbicide excipients can greatly increase susceptibility to genital herpes transmission in the mouse, BMC Infect Dis, 10 (2010) 331.

[27] K.H. Soergel, G.E. Whalen, J.A. Harris, Passive movement of water and sodium across the human small intestinal mucosa, J Appl Physiol, 24 (1968) 40-48.

[28] J. Fischbarg, Fluid transport across leaky epithelia: central role of the tight junction and supporting role of aquaporins, Physiol Rev, 90 (2010) 1271-1290.

[29] A.I. Masyuk, R.A. Marinelli, N.F. LaRusso, Water transport by epithelia of the digestive tract, Gastroenterology, 122 (2002) 545-562. 
[30] A.B. Noach, M. Sakai, M.C. Blom-Roosemalen, H.R. de Jonge, A.G. de Boer, D.D. Breimer, Effect of anisotonic conditions on the transport of hydrophilic model compounds across monolayers of human colonic cell lines, J Pharmacol Exp Ther, 270 (1994) 1373-1380.

[31] C.O. Billich, R. Levitan, Effects of sodium concentration and osmolality on water and electrolyte absorption form the intact human colon, J Clin Invest, 48 (1969) 1336-1347.

[32] K. Khanvilkar, M.D. Donovan, D.R. Flanagan, Drug transfer through mucus, Adv Drug Deliv Rev, 48 (2001) 173-193.

[33] H.H. Sigurdsson, J. Kirch, C.M. Lehr, Mucus as a barrier to lipophilic drugs, Int J Pharm, 453 (2013) 56-64.

[34] A. Carballo-Dieguez, J.A. Bauermeister, A. Ventuneac, C. Dolezal, I. Balan, R.H. Remien, The use of rectal douches among HIV-uninfected and infected men who have unprotected receptive anal intercourse: implications for rectal microbicides, AIDS Behav, 12 (2008) 860-866. [35] L. Misegades, K. Page-Shafer, D. Halperin, W. McFarland, Y.W.S.S.I.G.Y.W.s. Survey, Anal intercourse among young low-income women in California: an overlooked risk factor for HIV?, AIDS, 15 (2001) 534-535.

[36] S. Brody, J.J. Potterat, Assessing the role of anal intercourse in the epidemiology of AIDS in Africa, Int J STD AIDS, 14 (2003) 431-436.

[37] UNAIDS, AIDSInfo 2011, in.

[38] M.C. Boily, R.F. Baggaley, L. Wang, B. Masse, R.G. White, R.J. Hayes, M. Alary, Heterosexual risk of HIV-1 infection per sexual act: systematic review and meta-analysis of observational studies, Lancet Infect Dis, 9 (2009) 118-129.

[39] W.D. Mosher, A. Chandra, J. Jones, Sexual Behavior and Selected Health Measures: Men and Women 15-44 Years of Age, United States, 2002 in: Advance Data from Vital and Health Statistics, Center for Disease Control, 2005, pp. 1-56.

[40] C.W. Geary, E.A. Bukusi, Women and ARV-based HIV prevention - challenges and opportunities, J Int AIDS Soc, 17 (2014) 19356.

[41] I. McGowan, An overview of antiretroviral pre-exposure prophylaxis of HIV infection, Am J Reprod Immunol, 71 (2014) 624-630.

[42] C.S. Dezzutti, E.R. Brown, B. Moncla, J. Russo, M. Cost, L. Wang, K. Uranker, R.P. Kunjara Na Ayudhya, K. Pryke, J. Pickett, M.A. Leblanc, L.C. Rohan, Is wetter better? An evaluation of over-the-counter personal lubricants for safety and anti-HIV-1 activity, PLoS One, 7 (2012) e48328. 
Graphical abstract

Hypotonic delivery improves MPP distribution while causing no detectable epithelial damage Hypotonic Hypertonic

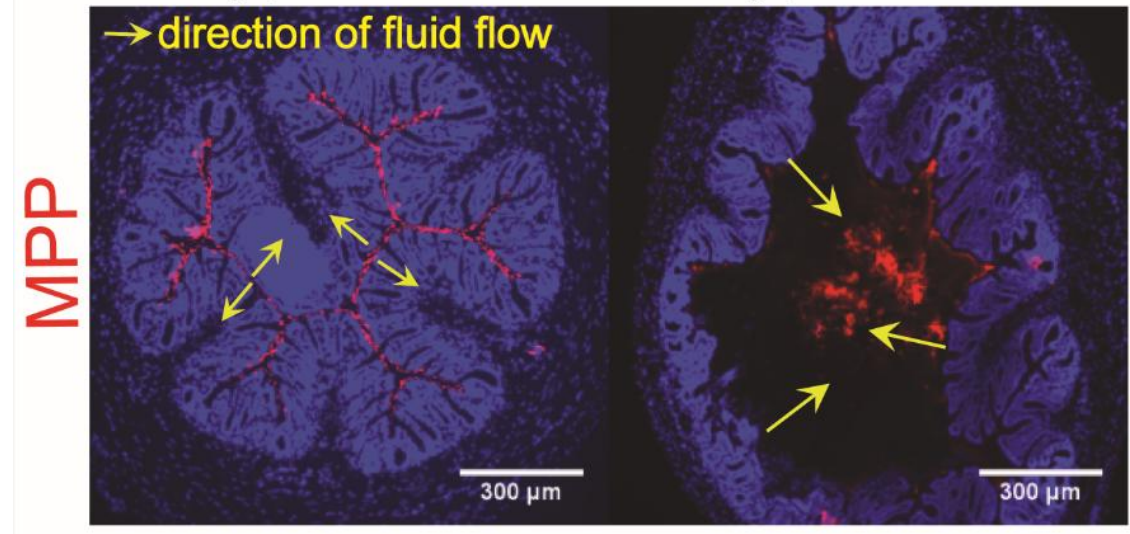

